\title{
AGB STAR MODELS
}

\author{
David Hollowell \\ Los Alamos National Laboratory \\ P.O. Box 1663, MS B220 \\ Los Alamos NM 87545
}

\begin{abstract}
Asymptotic giant branch star models are reviewed in relation to luminosity observations and chemical abundance observations of these stars.
\end{abstract}

\section{Review}

Asymptotic giant branch (AGB) star models aid in understanding AGB chemical evolution both directly (e.g., in M stars, S stars, and carbon stars) and indirectly (e.g, in barium stars, in the Sun, and in meteorites). An AGB star is noted for cyclic hydrogen ( $\mathrm{H}$ ) and helium (He) burning around a carbon-oxygen (C-O) degenerate core (e.g. Iben and Renzini, 1983). For $10 \%$ of a $\mathrm{H} / \mathrm{He}$ burning cycle, He burns quiescently, producing the model surface luminosity $\left(1000-10000 \mathrm{~L}_{\odot}\right)$, and dumping $\mathrm{C}$ and $\mathrm{O}$ onto the degenerate core. Helium burning in this shell is slowly extinguished, causing overlying $\mathrm{H}$-rich regions to contract and heat. Hydrogen begins to burn in a shell, and for $90 \%$ of the $\mathrm{H} / \mathrm{He}$ burning cycle, this produces a model surface luminosity (still $1000-10000 \mathrm{~L}_{\odot}$ ). This $\mathrm{H}$ burning dumps $\mathrm{He}$ on top of the $\mathrm{C}-\mathrm{O}$ core. Eventually this "fresh" helium will burn, making outer layers expand and cool, including the hydrogen burning layer. Once again, hydrogen burning will be extinguished, while helium buming provides the stellar luminosity.

Because of the compactness of the $\mathrm{C}-\mathrm{O}$ degenerate core, the ignition of helium occurs around the core in a thermal runaway, and for a short time the helium-burning luminosity can reach $10^{8} \mathrm{~L}_{\mathrm{O}}$. The energy in this luminosity pulse is largely absorbed by the expansion of interior regions, with excess energy being transported toward the stellar surface, through an interior shell of turbulent convection. This convective shell material includes carbon $(10-20 \%$ by mass, produced as a triple-alpha byproduct) as well as isotopes irradiated by neutrons neutrons produced in the $10^{8} \mathrm{~K}$ temperatures at the base of the convective shell (e.g. Howard, et al., 1986 or Gallino, et al., 1988).

This convection in low-mass AGB star models can be strong enough to mix processed material from the interior to the stellar surface, and mix hydrogen-rich surface material in toward the interior. This hydrogen can then be captured by abundant ${ }^{12} \mathrm{C}$ nuclei, forming ${ }^{13} \mathrm{C}$ nuclei, which (via He capture) act as a neutron irradiation source during a subsequent pulse. In all high-mass AGB star models, neutron irradiation occurs via alpha capture on ${ }^{22} \mathrm{Ne}$ (a 
byproduct of He-burning with ${ }^{14} \mathrm{~N}$ ).

In all AGB stars the escaping radiation causes the ever present red-giant envelope to extend towards the stellar interior (signaling the "dredge-up" phase), and this can mix carbon to the stellar surface (converting the star from an $\mathrm{M}$ star into a $\mathrm{C}$ star). Observed populations of bright $M$ and C AGB stars can be explained (e.g. Renzini and Voli, 1981) in terms of dredge up and hydrogen burning in the AGB envelope, and neutron-capture nucleosynthesis may be explained in terms of the ${ }^{22} \mathrm{Ne}$ neutron source (e.g. Truran and Iben, 1977). Observed populations of the dimmer M and C AGB stars may be explained in terms of an appropriate match of stellar core mass, envelope mass, and metallicity (e.g. Lattanzio, 1986, Boothroyd and Sackmann, 1988, or Hollowell and Iben, 1989), and neutron capture nucleosynthesis may be explained in terms of the ${ }^{13} \mathrm{C}$ neutron source.

\section{References}

Boothroyd, A. I. and Juliana-Sackmann, I., 1988, Astrophys. J., 328, 671.

Hollowell, D. and Iben, I. I. Jr., 1989, Astrophys. J., 340, 966.

Howard, W. M., Mathews, G. J., Takahashi, K., and Ward, R. A., 1986, Astrophys. J., 309, 633.

Iben, I. I., Jr. and Renzini, A., 1983, Ann. Rev. of Astron. and Astrophys., 21, 271.

Gallino, R., Busso, M., Picchio, G., Raiteri, C. M., Renzini, A., 1988, Astrophys. J., 334, L45.

Lattanzio, J. C., 1986, Astrophys. J., 311, 708.

Renzini, A. and Voli, M., 1981, Astron. and Astrophys., 94, 175.

Truran, J. W. and Iben, I. I. Jr, 1977, Astrophys. J., 216, 797. 